\section{Check list of medicinal plants from Navapur, district Nandurbar (Maharashtra State), India}

\author{
Ajitkumar G. Jaiswal \\ Arts, Commerce and Science College Navapur, Dist. Nandurbar 425 418, Mabarashtra, India
}

\begin{abstract}
Nandurbar is known as predominantly tribal district in Maharashtra. More than $80 \%$ population of the district is tribal. While studding flora of medicinal plants of Navapur Area, Navapur taluka author collected one hundred and fifty-eight medicinal plants. In this communication these medicinal plants were listed.
\end{abstract}

Key words:

Medicinal Plants, Navapur, Nandurbar district

\author{
Received: \\ 05 May 2017 \\ Accepted: \\ 15 June 2017 \\ Published: \\ 17 June 2017 \\ *Corresponding Author \\ Ajitkumar G. Jaiswal \\ Arts, Commerce and \\ Science College Navapur, \\ Dist. Nandurbar 425 418, \\ Maharashtra, India \\ Email: \\ navapuracs \\ @gmail.com \\ Citation:
Jaiswal. A.G. (2017).
Check list of medicinal
plants from Navapur,
district Nandurbar
(Maharashtra State), India.
Journal of Medicinal
Botany, 1, 20-28. doi:
10.25081/jmb.2017.v1.53
}

\section{Introduction}

Nandurbar is known as predominantly tribal district in Maharashtra. More than $80 \%$ population of the district is of tribals. These tribals reside small Padas in the interior forest areas. These tribals mostly depend on the forest resources to fulfill their day-to-day basic needs. Traditionally they depend upon local herbal medicine man for the treatment of various diseases. These local medicines man known by various names as Viadu, Bahagat etc. This local medicine man knows very well about the medicinal properties of plants present in surrounding area. They have well preserved the valuable treasure of knowledge for generations. But encroachment of modern facilities has put at risk this valuable knowledge. Young generation seem to be indifferent to this rich medicinal heritage being on the verge of extinction. Author made the collection of medicinal plants that are found in the Navapur Area of the Navapur taluka. Author also made the list of the names of such plant in local language. Total 158 medicinal plants were listed.

\section{Methodology}

Specimens of medicinal plants were collected in different sessions from Navapur area of Navapur taluka of Nandurbar district. These specimens were preserved in the Department of Botany. Plants were identified with the help of standard flora and research articles available Bhattacharjee (2000), Kurian (2003), Lakshminarasimhan and Sharma (1991), Matthew (1988), Parker (1924), Zingare (2012). Along with botanical name and family of each plant name in vernacular language is also coated. In all 158 medicinal plants have been identified and their quantitative abundance in the forest area has also been therein listed (Table 1). During collection field diary was maintained. 
Table 1. List of medicinal plants, family, vernacular name and quantitative abundance.

\begin{tabular}{|c|c|c|c|c|}
\hline Sl. No. & Botanical Name & Family & $\begin{array}{l}\text { Local name in vernacular } \\
\text { Language }\end{array}$ & $\begin{array}{l}\text { Quantitative } \\
\text { abundance }\end{array}$ \\
\hline 01 & Prosopis spicigera Linn. & Fabaceae & Himbadi & $\mathrm{C}$ \\
\hline 02 & Ficus tsiela Rlees & Moraceae & Pimpri & $\mathrm{RC}$ \\
\hline 03 & Jatropha gossypifolia Linn. & Euphorbiaceae & Roturondo & $\mathrm{C}$ \\
\hline 04 & Zizyphus oenopila Mill & Rhamnaceae & Chambor & $\mathrm{C}$ \\
\hline 05 & Mentha viridis Linn. & Lamiaceae & Pudina & $\mathrm{C}$ \\
\hline 06 & Butea monosperma (Lam.) Taub & Fabaceae & Palash & $\mathrm{RC}$ \\
\hline 07 & Moringa concanensis Nimmo & Moringaceae & Kodwohevgo & $\mathrm{C}$ \\
\hline 08 & Convolvulus microphyllus Linn. & Convolvulaceae & Lepdi & VR \\
\hline 09 & Sesamum indicum Linn. & Pedaliaceae & Tili & $\mathrm{R}$ \\
\hline 10 & Gossypium herbaceum Linn. & Malvaceae & Kapasyo & $\mathrm{VC}$ \\
\hline 11 & Pueraria tuberose DC & Fabaceae & Aadkuvo & $\mathrm{RR}$ \\
\hline 12 & Clitoria biflora Dalzell & Fabaceae & Charo & $\mathrm{RR}$ \\
\hline 13 & Clitoria ternatea $\mathrm{L}$. & Fabaceae & Kudo pano & $\mathrm{C}$ \\
\hline 14 & Cissus quadrangularis & Vitaceae & Kandvel & $\mathrm{RR}$ \\
\hline 15 & Wrightia arborea $\mathrm{L}$. & Apocynaceae & Dudh-kudi & $\mathrm{RR}$ \\
\hline 16 & Actinopeteris radiate Bedd & Pteridophyte & Bhuitad & $\mathrm{RC}$ \\
\hline 17 & Rungia pectinata $\mathrm{L}$. & Acanthaceae & Pindi & $\mathrm{RC}$ \\
\hline 18 & Barleria gibsoni $\mathrm{L}$. & Acanthaceae & Suruchikadi & $\mathrm{VC}$ \\
\hline 19 & Pergularia daemia (Forssk.) Chiov. & Asclepidaceae & Vishv & $\mathrm{R}$ \\
\hline 20 & Tamarindus indica $\mathrm{L}$. & Fabaceae & Khate emli & $\mathrm{VC}$ \\
\hline 21 & Phyllanthus emblica L. & Phyllanthaceae & Avale & VC \\
\hline 22 & Vachellia nilotica (L.)P.J.Hurter \& Mabb & Fabaceae & & $\mathrm{RC}$ \\
\hline 23 & Crocus sativus L. & Iridaceae & Kesaro & $\mathrm{RC}$ \\
\hline 24 & Calatropis gigantean R.Br. & Asclepidaceae & Ruwandi & $\mathrm{VC}$ \\
\hline 25 & Salmalia malabarica Sch. Et. Endt. & Bombaceae & Hawari & $\mathrm{RC}$ \\
\hline 26 & Eclipta alba Haask & Asteraceae & Mako & $\mathrm{RC}$ \\
\hline 27 & Mucuna pruriens DC & Fabaceae & Khojlo & $\mathrm{RR}$ \\
\hline 28 & Celosia argentea Linn. & Amaranthaceae & Ukhrudo & $\mathrm{VC}$ \\
\hline 29 & Ricinus communis Linn. & Euphorbiaceae & Oondoo & $\mathrm{VC}$ \\
\hline 30 & Biophytum sensitivum DC & Oxalidaceae & Lajwalyo & $\mathrm{RR}$ \\
\hline 31 & Tinospora cordifolia Miers & Menispermaceae & Kambanya velgo & $\mathrm{VC}$ \\
\hline 32 & Melia azedarch Linn. & Meliaceae & Bakanyo & $\mathrm{C}$ \\
\hline 33 & Momordica charantia Linn. & Cucurbitaceae & Karle & $\mathrm{RC}$ \\
\hline 34 & Urginea indica Kunth & Liliaceae & Janglikando & $\mathrm{C}$ \\
\hline 35 & Hibiscus esculentus Linn. & Malvaceae & Pende & $\mathrm{C}$ \\
\hline 36 & Acacia catechu Willd & Fabaceae & Kati & $\mathrm{RC}$ \\
\hline 37 & Citrus medica Linn. & Rutaceae & Nimbavi & $\mathrm{C}$ \\
\hline 38 & Argemone mexicana Linn. & Papaveraceae & Unahylo & $\mathrm{VC}$ \\
\hline 39 & Cissus quadrangularis Linn. & Vitaceae & Lagwel & $\mathrm{RR}$ \\
\hline 40 & Coriandrum sativum Linn. & Apiaceae & Khonthbhuro & $\mathrm{VC}$ \\
\hline 41 & Annona squamosa Linn. & Annonaceae & Satifol & $\mathrm{RR}$ \\
\hline 42 & Holarrhena antidysenterica Wall & Apocynaceae & Doodkavdo & $\mathrm{RR}$ \\
\hline 43 & Solanum xantocarpum Schrad \& Wendl & Solanaceae & Ringno & $\mathrm{VC}$ \\
\hline 44 & Ailanthus excels Roxb & Simaroubaceae & Worulo & $\mathrm{C}$ \\
\hline 45 & Cordia dichotoma Forst & Boraginaceae & Hetli & $\mathrm{RC}$ \\
\hline 46 & Carica papaya Linn. & Caricaceae & Herun kakdi & $\mathrm{C}$ \\
\hline 47 & Lawsonia inermis Linn. & Lythraceae & Mehadi & $\mathrm{RR}$ \\
\hline 48 & Terminalia belerica Roxb. & Combretaceae & Bedo & $\mathrm{VC}$ \\
\hline 49 & Vitex negundo Linn. & Lamiaceae & Nirgudi & $\mathrm{VC}$ \\
\hline 50 & Coleus aromaticus Benth & Lamiaceae & Pan-ajma & $\mathrm{VC}$ \\
\hline 51 & Vigna trilobata Verde & Fabaceae & Merchi kand & $\mathrm{RC}$ \\
\hline 52 & Triumfetta rotundifolia Lam. & Tiliaceae & Chikatyo & $\mathrm{RR}$ \\
\hline 53 & Adhatoda vasica Nees & Acanthaceae & Ardusoo & $\mathrm{VC}$ \\
\hline 54 & Commelina benghalensisi Linn. & Commelinaceae & Kenyo & $\mathrm{VC}$ \\
\hline 55 & Pongamia pinnata Pierre & Fabaceae & Konji & VC \\
\hline
\end{tabular}




\begin{tabular}{|c|c|c|c|c|}
\hline 56 & Ficus religiosa Linn. & Moraceae & Piplo & $\mathrm{C}$ \\
\hline 57 & Tridex procumbens Linn. & Asteraceae & Kodkya khod & VC \\
\hline 58 & Achyranthes aspera Linn. & Amaranthaceae & Chinjudo & VC \\
\hline 59 & Gardenia turgida Roxb. & Rubiaceae & Gungudo & VC \\
\hline 60 & Syzygium cumini Skeels & Myrtaceae & Hori & RR \\
\hline 61 & Phyllanthus niruri L. & Phyllanthaceae & Bhui avli & $\mathrm{C}$ \\
\hline 62 & Ischaemum pilosum Wight & Poaceae & Khoo & RR \\
\hline 63 & Withania somnifera Dunal & Solanaceae & Dolaro & $\mathrm{RC}$ \\
\hline 64 & Cucumis trigonus Roxb & Cucurbitaceae & Hapkachro & RR \\
\hline 65 & Musa paradisiaca Linn. & Musaceae & Kelo & $\mathrm{RC}$ \\
\hline 66 & Aegle marmelos Correa & Rutaceae & Bili & $\mathrm{C}$ \\
\hline 67 & Zingiber officinale Rose & Zingiberaceae & Aadrek & RR \\
\hline 68 & Mangifera indica Linn. & Anacardiaceae & Ambo & $\mathrm{C}$ \\
\hline 69 & Tribulus terrestris Linn. & Zygophyllaceae & Gokhro & $\mathrm{C}$ \\
\hline 70 & Limonia acidissima L. & Rutaceae & Kawthi & RR \\
\hline 71 & Abrus precatorius Linn. & Fabaceae & Tingle & $\mathrm{C}$ \\
\hline 72 & Baubinia racemosa Lam & Fabaceae & Hanglo & $\mathrm{C}$ \\
\hline 73 & Ocimum basillicum Linn. & Lamiaceae & Morvo & VC \\
\hline 74 & Aloe barbadensis Mill & Liliaceae & Detki & VC \\
\hline 75 & Tephrosia villosa Pers & Fabaceae & Unhali & RR \\
\hline 76 & Corchorus fascicularis Linn & Malvaceae & Hano chiktiyo & RR \\
\hline 77 & Cynodon dactylon Pers & Poaceae & Dorudo & RR \\
\hline 78 & Boerhaavia chinensis Aschers & Nyctaginaceae & Dogodfodyo & VR \\
\hline 79 & Cyperus rotundus Linn & Cyperaceae & Gundyo & VR \\
\hline 80 & Kalanchoe pinnata Pers & Crassulaceae & Lagpan & RR \\
\hline 81 & Caesalpinia crista Linn. & Fabaceae & Kachko & $\mathrm{C}$ \\
\hline 82 & Martynia annua Linn. & Martyniaceae & Waghnokhyo & $\mathrm{C}$ \\
\hline 83 & Cymbopogon citrates Stapf & Poaceae & Govati chha & $\mathrm{C}$ \\
\hline 84 & Cocculus birsutus Diels & Menispermaceae & Vasano & RR \\
\hline 85 & Ocimum sanctum Linn. & Lamiaceae & Lali tulsi & $\mathrm{C}$ \\
\hline 86 & Ocimum gratissimum Linn. & Lamiaceae & Ran tulsi & $\mathrm{C}$ \\
\hline 87 & Xanthium stromarium Linn. & Asteraceae & Cholyo & $\mathrm{RC}$ \\
\hline 88 & Enicostemma littorale Blume & Gentianaceae & Kodvinay & $\mathrm{C}$ \\
\hline 89 & Cassia tora Linn. & Fabaceae & Hano povadyo & RR \\
\hline 90 & Delonix elata Gamble & Fabaceae & Honuro & VR \\
\hline 91 & Barleria prionitis Linn. & Acanthaceae & Katchiri & $\mathrm{C}$ \\
\hline 92 & Dolichandrone falcate Seem & Bignoniaceae & Menhingi & $\mathrm{RC}$ \\
\hline 93 & Sorghum vulgare Pers & Poaceae & Hybridjuvha & $\mathrm{C}$ \\
\hline 94 & Psoralea corylifolia Linn. & Fabaceae & Bapchyo & $\mathrm{RC}$ \\
\hline 95 & Vicoa indica DC & Asteraceae & Mukhra & $\mathrm{RC}$ \\
\hline 96 & Dalbergia latifolia Roxb & Fabaceae & Chichmo & $\mathrm{C}$ \\
\hline 97 & Clerodendron phlomidis Linn. & Lamiaceae & Orni & RR \\
\hline 98 & Punica granatum Linn. & Lythraceae & Dalimbo & $\mathrm{C}$ \\
\hline 99 & Cassia auriculata Linn. & Fabaceae & Chamar avli & $\mathrm{C}$ \\
\hline 100 & Eleusine coracana Gaertn & Poaceae & Nagli & $\mathrm{C}$ \\
\hline 101 & Cleome viscosa Linn. & Cleomaceae & Bero & $\mathrm{VC}$ \\
\hline 102 & Hibiscus rosa-sinensis Linn. & Malvaceae & Jaswandi & $\mathrm{C}$ \\
\hline 103 & Luffa acutangula Roxb & Cucurbitaceae & Kodvo dotko & $\mathrm{C}$ \\
\hline 104 & Cajanus cajan Mill & Fabaceae & Tuvi & $\mathrm{C}$ \\
\hline 105 & Bryonopsis lanciniosa $\mathrm{Naud}$ & Cucurbitaceae & Kabrodovo & $\mathrm{RC}$ \\
\hline 106 & Trichodesma indicum R.Br. & Boraginaceae & Agyokhod & $\mathrm{RC}$ \\
\hline 107 & Holoptelea integrifolia Planch & Ulmaceae & Papdyo & $\mathrm{R}$ \\
\hline 108 & Terminalia arjuna W.\& A. & Combretaceae & Bhuro hojudo & $\mathrm{C}$ \\
\hline 109 & Ficus recemosa Linn. & Moraceae & Umbro & $\mathrm{R}$ \\
\hline 110 & Operculina turpethum Sliva & Convolulaceae & Tindari & $\mathrm{R}$ \\
\hline 111 & Uraria picta Desv & Fabaceae & Vandrorshepteyo & RR \\
\hline 112 & Stereospermum suaveolens DC & Verbenaceae & Padlo & $\mathrm{RC}$ \\
\hline 113 & Rungia repens $\mathrm{Nees}$ & Acanthaceae & Zunjoo & $\mathrm{RC}$ \\
\hline 114 & Madbuca indica Roxb & Sapotaceae & Mov & $\mathrm{C}$ \\
\hline 115 & Cymbopogon martinii & Poaceae & Rohiso & RR \\
\hline
\end{tabular}




\begin{tabular}{|c|c|c|c|c|}
\hline 116 & Orthosiphon pallidus Royle & Lamiaceae & Sonyo & RR \\
\hline 117 & Cuscuta reflexa Roxb & Convolvulaceae & Devoatte & $\mathrm{RR}$ \\
\hline 118 & Ficus hispida Linn. & Moraceae & Tedgi umbari & $\mathrm{RC}$ \\
\hline 119 & Psidium guajava Linn. & Myrtaceae & Jam & $\mathrm{C}$ \\
\hline 120 & Hemidesmus indicus R.Br. & Apocynaceae & Adgudi & RR \\
\hline 121 & Coccinia indica W \& A & Cucurbitaceae & Kodvo todlo & $\mathrm{RC}$ \\
\hline 122 & Allium sativum Linn. & Amarylidaceae & Gondalo & $\mathrm{C}$ \\
\hline 123 & Allium cepa Linn. & Amarylidaceae & Kando & $\mathrm{C}$ \\
\hline 124 & Lagenaria vulgaris Seringe & Cucurbitaceae & Dudiyo & VR \\
\hline 125 & Phaseolus trilobus Ait & Fabaceae & Ranmugyo & $\mathrm{C}$ \\
\hline 126 & Aristolocbia bracteata Retz & Aristolochiaceae & Geedan & $\mathrm{RC}$ \\
\hline 127 & Solanum verbascifolium Linn. & Solanaceae & Ranvengo & $\mathrm{RR}$ \\
\hline 128 & Cucurbita maxima Duch & Cucurbitaceae & Kolo & $\mathrm{C}$ \\
\hline 129 & Cyamopsis tetragonoloba Taub & Fabaceae & Gorwaya & VR \\
\hline 130 & Baliospermum montanum Muell & Euphorbiaceae & Datyo & $\mathrm{R}$ \\
\hline 131 & Apluda mutica Linn. & Poaceae & Tilkhodo & $\mathrm{RR}$ \\
\hline 132 & Crataeva nurvala Ham & Capparidaceae & Yayro & $\mathrm{RC}$ \\
\hline 133 & Morinda tinctoria Roxb & Rubiaceae & Aali & $\mathrm{RC}$ \\
\hline 134 & Azadirachata indica A.Juss & Meliaceae & Nimbo & $\mathrm{C}$ \\
\hline 135 & Costus speciosus Smith & Costaceae & Pevo & $\mathrm{RC}$ \\
\hline 136 & Tectona grandis Linn. & Verbanaceae & Hag & $\mathrm{C}$ \\
\hline 137 & Woodfordia fruticosa Kurz & Lythraceae & Pecheri & $\mathrm{C}$ \\
\hline 138 & Echinops echinatus Roxb & Asteraceae & Ubkatyo & $\mathrm{VC}$ \\
\hline 139 & Cressa cretica Linn. & Convolvulaceae & Ranchano & $\mathrm{VC}$ \\
\hline 140 & Solanum lycopersicum Linn. & Solanaceae & Tamato & $\mathrm{VC}$ \\
\hline 141 & Cucumis melo Linn. & Cucurbitaceae & Chibado & $\mathrm{C}$ \\
\hline 142 & Corchorus capsularia Linn & Malvaceae & Ranchucho & $\mathrm{RC}$ \\
\hline 143 & Asteracantha longifolia Nees & Acanthaceae & Agnikatyo & $\mathrm{RC}$ \\
\hline 144 & Boswellia serrata Roxb. & Burseraceae & Gugulo & VVR \\
\hline 145 & Hibiscus cannabinus Linn. & Malvaceae & Khato pendo & $\mathrm{VC}$ \\
\hline 146 & Portulaca oleraceae Linn. & Portulaceae & Khatgolo & $\mathrm{VC}$ \\
\hline 147 & Trichosanthes palmata Roxb. & Cucurbitaceae & Rankharbujo & $\mathrm{R}$ \\
\hline 148 & Grewia tiliaefolia Vahl. & Malvaceae & Tamno & $\mathrm{RR}$ \\
\hline 149 & Erythrina variegatata Linn. & Fabaceae & Pangro & $\mathrm{RR}$ \\
\hline 150 & Garuga pinnata Roxb. & Burseraceae & Kakud & $\mathrm{RC}$ \\
\hline 151 & Polycarpoea corymbosa Lam. & Caryophyllaceae & Pandhriphuli & $\mathrm{RR}$ \\
\hline 152 & Cassia fistula Linn. & Fabaceae & Cerwalo & $\mathrm{C}$ \\
\hline 153 & Erythrina corallodendron $\mathrm{L}$. & Fabaceae & Lal phulo & $\mathrm{RC}$ \\
\hline 154 & Aloe vera $(\mathrm{L})$ Burm.f. & Asphodelaceae & Korpad & $\mathrm{VC}$ \\
\hline 155 & Asparagus racemosus Willd & Asparagaceae & Shatur & $\mathrm{RC}$ \\
\hline 157 & Cassia fistula $\mathrm{L}$. & Fabaceae & Cha & $\mathrm{RR}$ \\
\hline 158 & Pomgamea pinnata L. & Fabaceae & Gro pattoo & $\mathrm{C}$ \\
\hline
\end{tabular}

Abbreviations used for quantitative abundance:

C-Common, RC-Rather Common, VC-Very Common, R-Rare, RR-Rather Rare, VR-Very Rare, VVR-Very Very Rare.

\section{Results}

In all 158 medicinal plants were identified from the study area. This finding will certainly help to make further research in the field of medicine, pharmacognosy and ethno botany.

\section{References}

Bhattacharjee, S. K. (2000). Handbook of aromatic plants, Pointer Pub., Jaipur.

Kurian, J. C. (2003). Plants that heal; Oriental Watchman Pub. House Pune.

Lakshminarasimhan, P. \& Sharma, B. D. (1991). Flora of Nasik district; BSI, New Delhi.
Matthew, K. M. (1988). Further Illustrations on the flora of the Tamilnadu Carnatic; The Rapinat Herbarium, St. Joseph's College Tiruchirapalli.

Parker, R. N. (1924). A forest flora for the Punjab with Hazara \& Delhi (Second Edition); Govt. Printing, Punjab.

Zingare, A. K. (2012). Encyclopedia of medicinal flora (Vol I \& II); Satyam Pub. \& Distributors, Jaipur. 